\title{
Screening for Frontotemporal Dementias and Alzheimer's Disease with the Philadelphia Brief Assessment of Cognition: A Preliminary Analysis
}

\author{
David J. Libon ${ }^{a}$ Lauren Massimo $^{b}$ Peachie Moore $^{b} \quad$ H. Branch Coslett ${ }^{b}$ \\ Anjan Chatterjee $^{b}$ Geoffrey K. Aguirre ${ }^{b}$ Aaron Rice $^{b}$ Luisa Vesely ${ }^{b}$ \\ Murray Grossman ${ }^{b}$ \\ ${ }^{a}$ New Jersey Institute for Successful Aging, University of Medicine and Dentistry of New Jersey, \\ School of Osteopathic Medicine, Stratford, N.J., and ${ }^{\mathrm{b}}$ Department of Neurology, University of Pennsylvania, \\ Philadelphia, Pa., USA
}

\section{Key Words \\ Alzheimer's disease • Frontotemporal dementia • \\ Philadelphia Brief Assessment of Cognition • \\ Neuropsychological assessment}

\begin{abstract}
Background: A neuropsychological screening instrument sensitive to neuropsychological deficits associated with Alzheimer's disease (AD) and patients with frontotemporal dementia (FTD) would be valuable for diagnostic evaluation. Methods: The Philadelphia Brief Assessment of Cognition (PBAC) assesses working memory/executive control, language, visuospatial operations, verbal/visual episodic memory, and behavior/social comportment and can be administered and scored in 15-20 min. Participants included 149 patients with AD and four groups of FTD patients - i.e., patients with a decline in social comportment, personality, and executive functioning (SOC/EXEC), semantic dementia (SemD), progressive nonfluent aphasia (PNFA), and corticobasal syndrome (CBS). Results: The total PBAC score corre-
\end{abstract}

A portion of this work was presented at the 5 th Frontotemporal Dementia Conference, San Francisco, Calif., USA.

\section{KARGER \\ Fax +41 613061234 \\ E-Mail karger@karger.ch}

(c) 2007 S. Karger AG, Basel $1420-8008 / 07 / 0246-0441 \$ 23.50 / 0$

www.karger.com
Accessible online at:

www.karger.com/dem lated with the Mini-Mental State Examination. Betweengroup analysis of PBAC subscales and the results of logistic regression analyses produced substantial between-group differences, emphasizing the sensitivity of the test to differentiate dementia subtypes. AD patients were impaired on tests of episodic memory, SOC/EXEC patients were impaired on a measure of social comportment/behavioral disturbance, PNFA patients obtained low scores on tests of working memory/executive control, SemD patients obtained lower scores on language-mediated measures, and CBS patients were impaired on visuospatial/visual memory tests. Conclusion: These data support the usefulness of the PBAC as a relatively brief screening test of overall dementia severity across a wide range of dementia patients.

Copyright $\odot 2007$ S. Karger AG, Basel

\section{Introduction}

Frontotemporal dementia (FTD) is a progressive neurodegenerative condition associated with imaging and pathological evidence of frontal and temporal lobe disease [1-3]. Clinical subgroups of patients with FTD have been described, including a decline in social comportment, personality, and executive functioning (SOC/

David J. Libon, $\mathrm{PhD}$

New Jersey Institute for Successful Aging, University of Medicine and Dentistry of New Jersey, School of Osteopathic Medicine, Suite 1800, 42 East Laurel Rd. Stratford, NJ 08084 (USA)

Tel. +1 215817 7708, E-Mail libondj@gmail.com 
EXEC); a fluent form of progressive aphasia associated with impaired word comprehension and poor object knowledge known as semantic dementia (SemD); a nonfluent aphasic syndrome associated with effortful speech and grammatical comprehension difficulty known as progressive nonfluent aphasia (PNFA), and a disorder of limb praxis, visuospatial processing, and executive functioning known as corticobasal syndrome (CBS).

Brief clinical measures such as the Mini-Mental State Examination (MMSE) are often used to assess disease severity in these patients. However, this instrument is relatively insensitive to many of the clinical features associated with these clinical syndromes as demonstrated in clinicopathological studies distinguishing Alzheimer's disease (AD) from FTD $[1,4,5]$. Thus, the MMSE may not be valid for gauging the overall severity or monitoring the clinical course of non-AD dementia patients. A wide number of tests exist to screen for cognitive impairment in dementia [6]. Other brief neuropsychological and behavioral assessment instruments that have been used to distinguish FTD from AD include the Frontal Assessment Battery (FAB) [7], the Addenbrooke Cognitive Examination (ACE) [8], the Neuropsychiatric Inventory [9], and the Frontal Behavioral Inventory [10]. Using brief neuropsychological protocols other researchers have shown that AD and FTD patients can be differentiated on the basis of their performance on subtests from the Wechsler Adult Intelligence Scale [11] and output obtained from tests of letter fluency $[7,12,13]$. However, much of this research has focused on the differentiation of $\mathrm{AD}$ from mixed groups of patients diagnosed with various FTD phenotypes.

Recently, a lengthy neuropsychological protocol has been shown to differentiate AD from FTD, and to distinguish among the four commonly mentioned FTD subgroups described above [14]. On the basis of this previous work and other research that has employed comprehensive neuropsychological protocols [15] we developed a brief neuropsychological screening instrument called the Philadelphia Brief Assessment of Cognition (PBAC). The $\mathrm{PBAC}$ can be administered and scored in approximately 15-20 min. Five domains of functioning are assessed: working memory/mental search, visuospatial/visuoconstructional operations, language-related skills, verbal/visual episodic memory, and personality/behavior. In the current research, the PBAC was administered to a consecutive series of patients with dementia due to $\mathrm{AD}$ and patients presenting with FTD.

The sensitivity of the PBAC to overall dementia severity was measured by correlating the total PBAC score with the MMSE. Thus, we predicted that the PBAC would be highly correlated with the MMSE, providing evidence that the PBAC can assess overall dementia severity. The sensitivity of the PBAC to various patterns of cognitive impairment seen in the dementia groups was also studied. Unlike the MMSE, we predicted that the PBAC will be sensitive to a wide variety of cognitive and/or behavioral problems associated with $\mathrm{AD}$ and the FTD phenotypes listed above.

\section{Methods}

\section{Patients}

Two hundred and three consecutive participants took part in this research. All patients were evaluated and recruited by experienced behavioral neurologists (G.K.A., A.C., H.B.C., M.G.) from the Department of Neurology, University of Pennsylvania, Philadelphia, Pa., USA. Patients were excluded if they were diagnosed clinically with mild cognitive impairment $(n=23)$, vascular dementia $(\mathrm{n}=11)$, amyotrophic lateral sclerosis $(\mathrm{n}=2)$, an atypical presentation of $\mathrm{AD}$ (posterior cortical atrophy syndrome, $\mathrm{n}=4$ ), a nonprogressive language disorder $(n=6)$, and diffuse Lewy body dementia $(n=8)$. These patient groups were not included in the current research for two reasons. First, the current research was undertaken to assess for differences between AD and FTD patients. Second, the small number of patients in most of the groups would make statistical comparisons difficult. This research was approved by the University of Pennsylvania Institutional Review Board and consent was obtained consistent with the Declaration of Helsinki.

The subgroups were classified based on previously published criteria [16-19] that have been modified to improve reliability. Subgroup classification was also based on previous research demonstrating unique radiological and pathological findings associated with each patient group $[1,4,5]$. At least two trained reviewers of a consensus committee confirmed the presence of specific diagnostic criteria and also assigned patients to an FTD subgroup based on an independent review of the semi-structured history obtained from patients and their families and a detailed neurologic examination. Interrater reliability for clinical diagnosis was 0.83 . The PBAC was not used as part of the diagnostic decisionmaking process. When there was disagreement between reviewers, the case was discussed by the entire committee to arrive at a consensus diagnosis.

Among the participants in this study, 108 patients were clinically diagnosed with FTD. Our sample included 49 patients with an SOC/EXEC profile. These patients presented with significant social and personality difficulties, often with alterations of executive functioning. Our sample also included 11 PNFA patients. These patients had effortful speech that may be associated with speech errors, phonemic substitutions, or impaired grammatical comprehension, but relatively good single-word comprehension. The sample also included 19 patients diagnosed with SemD. The disorder of these patients was characterized by fluent and circumlocutory spontaneous speech that was often empty in content with a prominent naming deficit and an associated deficit understanding single words and objects. Finally, 29 patients were diagnosed with CBS based on criteria derived from clinicopathologi- 
cal studies reported in the literature and our own autopsy series $[1,19]$. These patients had apraxia, visuospatial and/or language difficulty, and a lateralized extrapyramidal syndrome (e.g. unilateral limb rigidity, myoclonus, dystonia, alien hand phenomena). Another 41 patients were given a clinical diagnosis of $\mathrm{AD}$ based on National Institute of Neurologic and Communicative Disorders and Stroke-Alzheimer's Disease and Related Disorders Association criteria [20].

The initial clinical diagnosis of a neurodegenerative disease was consistent with the results of serum studies, structural imaging studies such as MRI or CT, studies of cerebrospinal fluid (when available), and functional neuroimaging studies such as SPECT or PET (these studies were not available to the consensus committee). Exclusion criteria included the presence of other neurologic conditions such as stroke or hydrocephalus (as determined by imaging studies reviewed by a neurologist), primary psychiatric disorders such as depression or psychosis, or a systemic illness that can interfere with cognitive functioning. Some of these patients were taking a fixed dosage of an acetylcholinesterase inhibitor (e.g. donepezil, rivastigmine, or galantamine). Some of these patients may also have been medicated with a low dosage of a nonsedating antidepressant (e.g. serotonin-specific reuptake inhibitors such as sertraline) or an atypical neuroleptic agent (e.g. quetiapine), as indicated clinically, but none of the patients demonstrated any evidence of sedation suggesting overmedication.

Table 1 summarizes the demographic features of these patients. Analysis of variance (ANOVA) indicated no differences in education or performance on the MMSE [21]. A between-group difference was present for age $\left(\mathrm{F}_{4,143}=11.03, \mathrm{p}<0.001\right)$. AD participants were older than patients diagnosed with SOC/EXEC $(\mathrm{p}<0.001)$ and CBS $(\mathrm{p}<0.001)$

\section{The Philadelphia Brief Assessment of Cognition}

The PBAC assesses 5 domains of neurobehavioral functioning which are critical to the assessment of dementia: working memo$\mathrm{ry} /$ mental search, language, visuospatial functioning, verbal/visual episodic memory, and social comportment/behavior. From the tests administered, 16 separate variables were scored. These 16 variables were grouped into the 5 PBAC subscales described below. The total PBAC score ranges between 0 and 126 .

The rationale for the construction of the 5 PBAC subscales was largely based on the results of previous research [14] where neuropsychological test performance of AD and FTD patients was subjected to a principal component analysis. On the basis of this research, latent variables were identified to measure the domains of neurobehavioral functioning described above. In our previous research [14], over $100 \mathrm{AD}$ and FTD patients were studied and patients were assigned to their respective diagnostic groups using the same methodology as described above. The principal component analysis yielded a 5-factor solution related to executive control, processing speed, lexical access, semantic memory, and episodic memory. An item analysis for each PBAC test within each of the 5 subscales was not conducted because of the limited number of individual test items and because of the unequal and sometimes small number of patients within some of the groups.

(1) Working Memory/Mental Search (Range 0-21). This scale contained 3 separate tests. Forward digit span (range 0-8) [22] was assessed with 6 trials consisting of 3-8 digits. Backward digit span (range 0-7) was assessed with trials consisting of 2-7 digits. Trials were administered until a patient failed 2 trials of the
Table 1. Demographic information (means \pm SD)

\begin{tabular}{llll}
\hline & Age & Education & MMSE \\
\hline AD & $73.37 \pm 9.55$ & $14.80 \pm 2.26$ & $19.42 \pm 6.74$ \\
SOC/EXEC & $61.96 \pm 8.83$ & $15.90 \pm 3.58$ & $23.07 \pm 8.19$ \\
PNFA & $63.45 \pm 8.91$ & $15.40 \pm 2.27$ & $23.78 \pm 4.94$ \\
SemD & $67.78 \pm 5.73$ & $15.11 \pm 2.58$ & $17.92 \pm 10.49$ \\
CBS & $63.90 \pm 8.08$ & $14.84 \pm 2.34$ & $21.93 \pm 5.32$ \\
\hline
\end{tabular}

same span length. The dependent variable for each test was the longest span correctly recalled. On a letter fluency test, patients were given $60 \mathrm{~s}$ to generate words, excluding proper nouns and numbers, beginning with a specified letter [23] (letter ' $F$ '). The dependent variable on this test was an ordinal scale (range 0-6) whereby zero response was assigned a score of $0 ; 1-3$ responses were assigned a value of $1 ; 4-6$ responses were assigned a value of 2; 7-9 responses were assigned a value of 3; 10-12 responses were assigned a value of $4 ; 13-15$ responses were assigned a value of 5 , and 16 or more responses were assigned a value of 6 .

(2) Language (Range 0-25). Five tests assessed language-related skills. Naming (range 0-6) was assessed by asking patients to name 6 specified objects present in the examining room. For sentence comprehension (range 0-9), 4 sentences were read to the patient (e.g. 'point to the desk and then point to the phone'). For each sentence, 2 responses were required. Sentence writing (range $0-3)$ was assessed by asking patients to produce a sentence about the weather. One point each was scored for adequate content, correct grammar, and the absence of any spelling errors. Conversational speech was scored clinically (range $0-3$ ). In order to keep the scaling of this subtest consistent with other PBAC subtests, half a point was subtracted from a score of 3 for word finding difficulty, circumlocution, semantic paraphasic errors, literal paraphasic errors, agrammatical speech, and effortful speech. Thus, if speech was normal, the patient earned 3 points. Word reading (range $0-4$ ) was assessed by asking patients to read 4 irregular words - choir, yacht, pint, cough.

(3) Visuospatial/Visuoconstructional Skills (Range 0-26). Three tests assessed visuospatial ability. Visuoconstruction ability was assessed by asking the patient to copy a modified version of the Rey Complex Figure (range 0-12) [24]. Line orientation (range 0-6) consisted of an array of 9 lines drawn to various angles. Below the array, 4 additional lines were also drawn to various angles, and patients matched each of these lines to a target in the non-line array. Praxis (range $0-8$ ) was assessed by asking patients to demonstrate how to use a hammer and scissors, and imitate the examiner when he placed the dorsum of his left hand against the right cheek, and then placed the dorsum of the left hand against the left cheek. Two points were assigned for the correct execution of each command. One point was assigned if the patient responded with a body-part-as-object response or required prompting for the nonmeaningful gestures.

(4) Episodic Memory (Range 0-30). Verbal memory and learning were assessed with a 6 -word list administered over 3 trials. Delayed free recall for this list was assessed after a filled delay. This was following by a delayed recognition test for the words that were not recalled. The stimuli consisted of the 6 original words 
Table 2. PBAC raw score of the composite indices and total score (means \pm SD)

\begin{tabular}{|c|c|c|c|c|c|}
\hline & $\mathrm{AD}$ & SOC/EXEC & PNFA & SemD & CBS \\
\hline $\begin{array}{l}\text { Working memory/ } \\
\text { mental search }\end{array}$ & $11.05 \pm 4.00$ & $11.35 \pm 4.47$ & $9.30 \pm 3.19$ & $7.00 \pm 4.74$ & $10.89 \pm 2.54$ \\
\hline Language & $15.83 \pm 4.75$ & $16.95 \pm 5.80$ & $17.00 \pm 4.04$ & $10.35 \pm 7.01$ & $15.65 \pm 4.72$ \\
\hline Visuospatial & $16.30 \pm 7.46$ & $19.22 \pm 6.06$ & $18.54 \pm 5.16$ & $17.27 \pm 8.20$ & $10.18 \pm 7.71$ \\
\hline Total episodic memory & $7.89 \pm 4.69$ & $16.85 \pm 8.74$ & $19.63 \pm 7.30$ & $12.19 \pm 8.83$ & $12.08 \pm 5.46$ \\
\hline Verbal memory & $6.72 \pm 3.45$ & $10.82 \pm 5.42$ & $12.63 \pm 5.25$ & $6.91 \pm 5.55$ & $9.93 \pm 3.27$ \\
\hline Visual memory & $1.41 \pm 2.32$ & $6.08 \pm 4.15$ & $7.00 \pm 3.80$ & $5.28 \pm 3.96$ & $2.16 \pm 3.12$ \\
\hline Behavior & $19.03 \pm 3.03$ & $14.25 \pm 3.76$ & $18.64 \pm 2.01$ & $16.44 \pm 3.25$ & $17.12 \pm 2.68$ \\
\hline Total score & $66.51 \pm 19.39$ & $78.37 \pm 24.91$ & $85.95 \pm 11.46$ & $63.08 \pm 27.66$ & $64.15 \pm 18.31$ \\
\hline
\end{tabular}

and 6 novel words that were semantically related to each of the targets. On the delayed recognition test, patients were asked to simply identify which words were on the original word list. One point was awarded for each word recalled freely, and half a point was given for recognition of a target word and rejection of the semantically related foil. Visual episodic memory was assessed by asking patients to reproduce a modified Rey Complex Figure after a 2-min filled delay (range 0-12). Four dependent variables were scored - number of words reproduced on the third learning trial of the word list (range 0-6), delayed verbal free recall (0-6) and performance on the delayed recognition test (range 0-6), and delayed Rey Complex Figure recall (range 0-12).

(5) Social Comportment and Behavior Scale (Range 0-24). Alterations in social comportment and behavior were assessed through a combination of interviewing a family member and observation of the patient's behavior during the clinical interview. Six behavioral domains were queried - apathy/poor initiation, disinhibition, social comportment, agitation/irritability, ritual/ obsessive compulsive behavior, and lack of empathy. Each behavioral symptom was scored on a 4 (not observed) to 1 (severe) ordinal scale.

\section{Results}

\section{PBAC Total Index}

The sensitivity of the PBAC to determine overall dementia severity was assessed by correlating the total PBAC score with the MMSE. Consistent with our prediction, the total PBAC score was highly correlated with the MMSE $(\mathrm{r}=0.885, \mathrm{p}<0.001)$. Although the 1-way ANOVA for the total PBAC score resulted in a significant main effect for group $\left(\mathrm{F}_{4,118}=3.77, \mathrm{p}<0.006\right)$, follow-up analyses revealed only borderline between-group differences such that PNFA patients obtained a marginally better score compared to SemD $(\mathrm{p}<0.079)$ and CBS $(\mathrm{p}<0.069)$ patients. CBS patients also obtained a slightly better score than SOC/EXEC patients $(\mathrm{p}<0.078)$.

\section{PBAC Composite Indices (Between-Group Analysis)}

The sensitivity of the PBAC to various patterns of neuropsychological impairment in patient groups was first examined by subjecting the raw data for each of the 5 composite indices to a 2 -way, group $\times$ domain repeatedmeasures ANOVA. This analysis yielded a main effect for neuropsychological domain of cognitive functioning $\left(\mathrm{F}_{4,115}=89.12, \mathrm{p}<0.001\right)$, and more importantly, a significant group $\times$ domain interaction $\left(\mathrm{F}_{16,454}=11.90\right.$, $\mathrm{p}<0.001)$. Second, raw data for the 5 composite indices were then analyzed with separate 1-way ANOVAs. These analyses resulted in a significant effect for group for all 5 composite indices (working memory/mental search: $\mathrm{F}_{4,141}=4.45, \mathrm{p}<0.002$; language: $\mathrm{F}_{4,133}=4.92, \mathrm{p}<0.001$; visuospatial: $\mathrm{F}_{4,135}=7.96, \mathrm{p}<0.001$; episodic memory: $\mathrm{F}_{4,139}=10.85, \mathrm{p}<0.001$; behavior: $\mathrm{F}_{4,135}=13.08, \mathrm{p}<$ 0.001 ). Covarying for age did not change these results (table 2).

Follow-up analyses were performed with Tukey tests. For the visuospatial index, CBS patients scored lower than AD ( $p<0.007)$, SOC/EXEC ( $<<0.001)$, PNFA ( $p<$ 0.009 and SemD $(p<0.009)$ patients. In the area of episodic memory, AD patients scored lower than SOC/EXEC $(\mathrm{p}<0.001)$ and PNFA patients $(\mathrm{p}<0.001)$. Also, CBS patients obtained a lower score compared to SOC/EXEC $(\mathrm{p}<0.041)$ and PNFA $(\mathrm{p}<0.028)$ patients. On the language index, SemD patients obtained a lower score compared to other patient groups (AD: $p<0.009$; SOC/EXEC: $\mathrm{p}<0.001$; PNFA: $\mathrm{p}<0.016$; CBS: $\mathrm{p}<0.014)$. SemD patients also scored low on the working memory/mental search index, compared to AD $(\mathrm{p}<0.004)$, SOC/EXEC, $(\mathrm{p}<0.001)$, and CBS $(\mathrm{p}<0.012)$ patients. For the social comportment/behavior scale, the SOC/EXEC patients exhibited a greater level of disturbance compared to all other groups ( $p<0.004$ for all analyses). 
Because CBS and SemD patients are known to present with modality-specific cognitive impairments [8, 19], separate analyses were conducted to assess for modalityspecific deficits on delayed verbal and delayed visual memory tests. One-way ANOVAs yielded significant main effects for group for verbal episodic memory $\left(\mathrm{F}_{4,144}=7.50, \mathrm{p}<0.001\right)$ and delayed visual memory (Rey Complex Figure; $\left.\mathrm{F}_{4,139}=14.11 \mathrm{p}<0.001\right)$. Follow-up analyses for delayed verbal memory test performance revealed that AD patients continued to score lower than SOC/ EXEC ( $p<0.001)$, PNFA ( $<<0.002)$, and CBS ( $p<0.034)$ patients. SemD patients also obtained lower scores compared to SOC/EXEC $(\mathrm{p}<0.019)$ and PNFA $(\mathrm{p}<0.011)$ patients. For delayed visual memory tests (Rey Complex Figure), AD patients obtained lower scores compared to SOC/EXEC $(\mathrm{p}<0.001)$, PNFA $(\mathrm{p}<0.001)$, and SemD $(p<0.002)$ patients. Now, CBS patients also scored lower on this test compared to SOC/EXEC ( $\mathrm{p}<0.001)$, PNFA $(\mathrm{p}<0.001)$, and SemD $(\mathrm{p}<0.028)$ patients.

\section{Multinomial Logistic Regression}

The sensitivity of the PBAC to various cognitive impairments was also assessed with a series of logistic regression analyses. A series of likelihood ratios was calculated using multinomial logistic regression analyses, guided by the results of the between-group analyses described above such that the reference group used for each logistic regression analysis (i.e., the group against which all other groups are compared) was based on the group that displayed the greatest impairment on any single PBAC index. For example, the AD group was the reference group for the PBAC total memory index. For this analysis $\left(\chi^{2}=38.80\right.$, d.f. $\left.=4, \mathrm{p}<0.001\right)$, the $\mathrm{AD}$ group was differentiated from all other patient groups $(\mathrm{p}<0.001)$. For the PBAC social comportment and behavior index $\left(\chi^{2}=46.38\right.$, d.f. $\left.=4, p<0.001\right)$, the SOC/EXE group was also differentiated from all other patient groups $(\mathrm{p}<$ $0.001)$. For the PBAC visuospatial index $\left(\chi^{2}=26.72\right.$, d.f. $=4, p<0.001)$, CBS patients were correctly classified and differentiated from all other patient groups $(\mathrm{p}<$ 0.007). Similarly, for the visual memory index $\left(\chi^{2}=47.24\right.$, d.f. $=4, p<0.001)$, CBS patients continued to be correctly classified and differentiated from all other groups $(\mathrm{p}<$ $0.007)$ except the AD group. For the PBAC language in$\operatorname{dex}\left(\chi^{2}=16.88\right.$, d.f. $\left.=4, p<0.002\right)$, SemD patients were differentiated from all other patient groups $(\mathrm{p}<0.004)$. Finally, for the PBAC working memory/mental search in$\operatorname{dex}\left(\chi^{2}=15.78\right.$, d.f. $\left.=4, p<0.003\right)$, SemD patients were differentiated from AD ( $\mathrm{p}<0.002)$, SOC/EXEC $(\mathrm{p}<$ $0.001)$, CBS ( $\mathrm{p}<0.004)$, but not PNFA patients.

The Philadelphia Brief Assessment of Cognition

\section{Discussion}

In the present research, we described a relatively brief assessment instrument that could be used within the context of a busy clinical practice. Unlike the MMSE, we sought to develop a test that is sensitive both to overall cognitive impairment and to the various cognitive/behavioral deficits that occur in patients with AD and FTD. The sensitivity of the PBAC to assess for overall dementia severity is supported by its robust correlation with the MMSE. The sensitivity of the PBAC to various patterns of neuropsychological impairment was assessed with the between-group and logistic regression analyses. AD patients displayed greater impairment on tests of episodic memory, SemD patients were disadvantaged on language and working memory/mental search tests, SOC/EXEC patients presented with greater behavioral problems, and CBS patients were differentially impaired on tests of visuospatial functioning and visual memory.

A variety of other screening instruments have been used to assess disease severity in AD and FTD. Using the FAB [4], researchers have been able to evaluate patients with AD and FTD. However, to our knowledge there is no research demonstrating that the $\mathrm{FAB}$ can differentiate between AD, CBS, and SemD. One of the FAB subtests requires patients to provide the superordinate concept for word pairs (similarities). The authors treat all erroneous responses as evidence of executive dysfunction. However, recent research $[25,26]$ has demonstrated a distinction between 'in-set' errors (dog-lion: 'you can own them') versus 'out-of-set' errors (dog-lion: 'one barks and the other growls'). Only out-of set errors have been shown to reflect actual executive impairment $[25,26]$.

The ACE [5] is another screening instrument used to evaluate patients with FTD. The ACE is essentially an elaboration on the MMSE. Research has shown that the ACE can assess AD and a mixed group of SOC/EXEC, SemD, and PNFA patients. Similar to the results reported above, AD patients obtained lower scores on tests of episodic memory, whereas FTD patients obtained lower scores on tests of letter fluency and naming as well as other language-related tests [5]. The ACE has also been shown to be sensitive to patients with mild AD compared to questionable $\mathrm{AD}$ [27] (i.e., $\mathrm{AD}$ and a mixed group of FTD patients) and patients suffering from major depression and AD [28].

The ACE has recently been revised [29]. Like the PBAC, domain subscales are now available. When a combined group of SOC/EXEC, SemD, and PNFA patients were compared to AD patients, a dissociation was found such

Dement Geriatr Cogn Disord 2007;24:441-447 
that $\mathrm{AD}$ patients obtained lower scores on tests assessing orientation and memory whereas the combined FTD group obtained lower scores on fluency and language. Another difference between the two tests is an assessment of social comportment/behavior which is part of the PBAC.

There are several shortcomings in the present research that need to be acknowledged and addressed in future research. First, the sensitivity of the PBAC to various patterns of neuropsychological impairment may be improved by including nonverbal working memory/mental search and semantic memory tests. In the current research, it was the SemD group who obtained the lowest score on the working memory/mental search index. The differential impairment seen in this group on this index may have been partially due to the verbal nature of the tests used to assess this domain of functioning [30]. Also, the inclusion of a specific test that measures semantic memory may improve the ability of the PBAC to differentiate between dementia groups.

Second, several statistical issues should be addressed in a revision of the PBAC including an item analysis of all individual PBAC tests. This would help identify a core set of measures that contribute to the total PBAC score and the various PBAC subscales, and allow us to shorten this measure. For example, we examined several subgroups of patients to evaluate the sensitivity of the PBAC to patients with different phenotypes. While this helped assess the sensitivity of this instrument, we were unable to assess specificity satisfactorily. Larger numbers of patients, the inclusion of patients with other phenotypes (i.e., vascular dementia, Lewy body dementia), and the evaluation of patients with autopsy-proven disease are necessary to improve the specificity of the PBAC for the purpose of differential diagnosis and ensure the validity of our observations.
Lastly, we acknowledge that for some FTD patients various cognitive as well as behavioral deficits can overlap. SemD patients frequently have a social disorder that is the hallmark of SOC/EXEC patients, even though the dominant impairment of SemD patients is their semantic impairment $[31,32]$. In the present study, the SOC/EXEC and PNFA patient groups both presented with impaired performance on tests of working memory/mental search. Executive limitations may contribute to the speech and language deficits of PNFA patients [33] to the extent limitations in working memory play a role in interpreting grammatically complex sentences. Likewise, executive limitations in SOC/EXEC patients may contribute to features of their social disorder. For example, an executive impairment of organization and planning can contribute to the appearance of apathy, and an executive deficit of inhibitory control may play a role in the disorder of social comportment seen in SOC/EXEC patients. The visual disorder associated with the clinical syndrome of CBS may resemble the phenotype of posterior cortical atrophy that is often caused by $\mathrm{AD}$ [34]. We do not intend to suggest that this relatively brief survey can provide a pathological diagnosis.

With these caveats in mind, the data described above support the usefulness of the PBAC. The PBAC provides a potentially efficient bedside assessment of overall dementia severity that is sensitive to the neuropsychological and behavioral indices that typify subgroups of patients with AD and non-AD dementia such as FTD.

\section{Acknowledgments}

This work was supported in part by the US Public Health Service (AG17586, AG15116, AG10124, and NS44266) and the Dana Foundation.

\section{References}

$\checkmark 1$ Forman MS, Farmer J, Johnson JK, Clark $\mathrm{CM}$, Arnold SE, Coslett HB, et al: Frontotemporal dementia: clinicopathological correlations. Ann Neurol 2006;59:952-962.

-2 Grossman M: Frontotemporal dementia: a review. J Int Neuropsychol Soc 2002;8:564583.

3 Snowden JS, Neary D, Mann DM: Frontotemporal Lobar Degeneration: Frontotemporal Dementia, Progressive Aphasia, Semantic Dementia, ed 1. New York, Churchill Livingstone, 1996.
4 Hodges JR, Davies RR, Xuereb J, Casey BJ, Broe M, Bak T, et al: Clinicopathological correlates in frontotemporal dementia. Ann Neurol 2004;56:399-406.

$\checkmark 5$ Knopman DS, Boeve BF, Parisi JE, Dickson DW, Smith GE, Ivnik RJ, et al: Antemortem diagnosis of frontotemporal lobar degeneration. Ann Neurol 2004;57:480-488.

6 Hutchinson AD, Mathias JL: Neuropsychological deficits in frontotemporal dementia and Alzheimer's disease: a meta-analytic review. J Neurol Neurosurg Psychiatry 2007; 78:917-928.
Dubois B, Slachevsky A, Litvan I, Pillon B: The FAB: a frontal assessment battery at bedside. Neurology 2000;55:1621-1626.

$\checkmark 8$ Mathuranath PS, Nestor PJ, Berrios GE, Rakowicz WP, Hodges JR: A brief cognitive test battery to differentiate Alzheimer's disease and frontotemporal dementia. Neurology 2000;55:1613-1620.

9 Cummings JL, Mega M, Gray K, RosenbergThompson S, Carusi DA, Gornbein J: The Neuropsychiatric Inventory: comprehensive assessment of psychopathology in dementia. Neurology 1994;44:2308-2314. 
10 Kertesz A, Nadkarni N, Davidson W, Thomas AW: The Frontal Behavioral Inventory in the differential diagnosis of frontotemporal dementia. J Int Neuropsychol Soc 2000;6: 460-468.

-11 Elfgren C, Brun A, Gustafson L, et al: Neuropsychological tests as discriminators between dementia of the Alzheimer's type. Int J Geriatr Psychiatry 1994;9:635-642.

-12 Pasquier F, Lebert F, Grymonprez L, Petit H: Verbal fluency in dementia of frontal lobe type and dementia of the Alzheimer's type. J Neurol Neurosurg Psychiatry 1995;58:8184.

13 Rascovsky K, Salmon DP, Hansen LA, Thal LJ, Galasko D: Disparate letter and semantic category fluency deficits in autopsy-confirmed frontotemporal dementia and $\mathrm{Alz}$ heimer's disease. Neuropsychology 2007;21: 20-30.

14 Libon DJ, Xie SX, Moore P, Farmer J, Antani S, McCawley G, et al: Patterns of neuropsychological impairment in frontotemporal dementia. Neurology 2007;68:369-375.

15 Kramer JH, Jurik J, Sha SJ: Distinctive neuropsychological patterns of frontotemporal dementia, semantic dementia, and Alzheimer's Disease. Cogn Behav Neurol 2003;16: 211-218.

16 Grossman M, Ash S: Primary progressive aphasia: a review. Neurocase 2004;10:3-18.

-17 McKhann G, Trojanowski JQ, Grossman M, Miller BL, Dickson D, Albert M: Clinical and pathological diagnosis of frontotemporal dementia: report of a work group on frontotemporal dementia and Pick's disease. Arch Neurol 2001;58:1803-1809.
18 Neary D, Snowden JS, Gustafson L, Passant U, Stuss D, Black S, et al: Frontotemporal lobar degeneration: a consensus on clinical diagnostic criteria. Neurology 1998;51:15461554.

19 Murray RC, Neumann M, Farmer J, Forman MS, Johnson JK, Miller BL, et al: Cognitive and motor assessment in autopsy-proven corticobasal degeneration. Neurology 2007; 68:1274-1283.

20 McKhann G, Drachman D, Folstein M, Katzman R, Price D, Stadian EM: Clinical diagnosis of Alzheimer's disease: report on the NINCDS-ADRDA work group under the auspices of the Department of Health and Human Services Task Force on Alzheimer's disease. Neurology 1984;34:939-944.

21 Folstein MF, Folstein SE, McHugh PR: 'Minimental state'. A practical method for grading the cognitive state of patients for the clinician. J Psychiatr Res 1975;12:189-198.

22 Wechsler D: Wechsler Adult Intelligence Scale - Revised. San Antonio, The Psychology Corporation, 1987.

23 Spreen O, Struss E: A Compendium of Neuropsychological Tests. New York, Oxford University Press, 1990.

24 Lezak M, Hanney J, Loring D: Neuropsychological Assessment, ed 3. New York, Oxford University Press, 2004.

25 Giovannetti T, Lamar M, Cloud BS, et al: Different underlying mechanisms for deficits in concept formation in dementia. Arch Clin Neuropsychol 2001;16:547-560.

26 Lamar M, Goldstein F, Libon DJ, Ashley AV, Lah JJ, Levey AI: The role of specific vascular biomarkers on executive functioning in $\mathrm{Alz}$ heimer's disease. 10th Int Conf on Alzheimer's Dis Relat Disord, Madrid, 2006.
7 Galton CJ, Erzinclioglu S, Sahakian BJ, Antoun N, Hodges JR: A comparison of Addenbrooke's Cognitive Examination (ACE), conventional neuropsychological assessment, and simple MRI-based medial temporal lobe evaluation in early diagnosis of Alzheimer's disease. Cogn Behav Neurol 2005;18:144150

28 Bak TH, Rogers TT, Crawford LM, Hearn VC, Mathuranath PS, Hodges JR: Cognitive bedside assessment in atypical parkinsonian syndromes. J Neurol Neurosurg Psychiatry 2005;76:420-422.

29 Mioshi E, Dawson K, Mitchell J, Arnold R, Hodges J: The Addenbrooke Cognitive Examination Revised (ACE-R): a brief cognitive screening battery for dementia screening. Int J Geriatr Psychiatry 2006;21: 1078-1085.

30 Luria AR: Higher Cortical Functions. New York, Basic Books, 1980.

31 Williams GB, Nestro PJ, Hodges JR: Neural correlates of semantic and behavioral deficits in frontotemporal dementia. Neuroimage 2005;24:1041-1051.

32 Edwards-Lee T, Miller BL, Benson DF, Cumminings JL, Russell GL, Boone K, Mena I: The temporal variant of frontotemporal dementia. Brian 1997;120:1027-1040.

33 Grossman M, Moore P, Koenig P, Antani S, McCawley G, Cross K, Kwok S: Semantic knowledge and categorization difficulty: a longitudinal study of Alzheimer's disease and frontotemporal dementia. Submitted.

34 Benson DF, Davis, RJ, Snyder BD: Posterior cortical atrophy. Arch Neurol 1988;45:789793. 